\section{Right patient, right time, right pharmacist: the time for clinical prioritisation tools?}

\author{
Penny Lewis
}

Hospital pharmacists play an essential and ever expanding role in patient care by ensuring that medicines are used safely and effectively. However, as pharmacists' roles have expanded, so, too, have patient numbers. Without a corresponding match in the number of hospital pharmacists, decisions have to be made about which patients should be provided clinical pharmacy services. Clinical pharmacy has evolved considerably over the past two decades, yet the approach to deploying clinical pharmacy services has generally remained unchanged. The traditional, and perhaps futile, approach of pharmacists working on a bed-by-bed basis to provide their services is being questioned. Does this conventional approach mean that patients who are at risk of adverse drug events can be overlooked by pharmacists by simply sitting in the final bed of a hospital ward? Are pharmacists reviewing patients on a daily basis who are unlikely to experience any harm from medicines? The answer to both of these questions is probably yes, with the former scenario likely to be of greater concern to pharmacists whose primary endeavour is the safety of patients. However, one cannot overlook the inefficiencies and costs generated by the second scenario.

Hospital pharmacists will know that the pharmaceutical complexity of patients is diverse and changeable. Those patients with complex or acute needs might benefit from daily review by a pharmacist with the appropriate skills and experience. There are also patients whose needs are minimal and the impact of a pharmacist intervention is small, and in this situation, we could be making better use of the

Correspondence to Dr Penny Lewis, Division of Pharmacy and Optometry, Manchester Academic Health Sciences Centre (MAHSC), University of Manchester, Manchester M13 9PT, UK; penny.lewis@manchester. ac.uk wider pharmacy team and the skills and knowledge of the technician workforce. Ultimately, the ability to screen and prioritise patients who need the most pharmacy input, and also those who do not, would clearly be of much benefit to patients and pharmacy teams.

One such prioritisation tool has been developed by Middlemore hospital in New Zealand. ${ }^{1}$ The Assessment of Risk Tool (ART) tool is a virtual real-time electronic tool that can stratify patients into those at high, medium or low risk for medication errors and adverse drug events. This system uses a number of risk flags, each with an associated weighted score, and then calculates a total score which corresponds to a particular risk group. This information is then used to prioritise pharmacist resources. There have been similar tools developed in the $\mathrm{UK}^{23}$ and which have been described in previous editions of the EJHP. ${ }^{3}$ Most tools are, however, developed locally using professional opinion, and robust validation of the effectiveness of tools is rare. Falconer and colleagues, in their paper included in this edition of EJHP, have taken this important next step and tested their tool to see if it does identify patients at risk of medication discrepancies during the admissions process and therefore those patients who would benefit from timely review by a pharmacist. The results are encouraging and highlight the contribution that particular risk factors such as polypharmacy and readmission have in relation to the risk of mediation-related problems.

Current economic pressures and a rise in demand for services mean that hospitals have to do more for less. With a need to seek out ways to improve efficiency while maintaining patient safety, locally developed prioritisation tools are seemingly on the rise. Furthermore, in the UK, this trend is likely to increase with National Health Service England actively recommending that hospitals provide targeted clinical services. ${ }^{4}$ However, what is clear is that there is disparity in approaches as well as in the extent that evidence is used to develop and test prioritisation tools.

Nursing is currently leading the way in this area having invested, developed and widely implemented prioritisation or acuity tools that classify the severity of a patient's condition and intensity of care that patient's will require. Such tools allow safe nursing levels to be calculated and ultimately aim to ensure that patients are seen by the right staff with the right skills in the right place. It is now time to bring pharmacy in line with nursing, but to do so, further research, such as that undertaken by Falconer et al, is required so that pharmacy can pursue the ambition of ensuring the right patient is being seen at the right time by the right pharmacist.

\section{Competing interests None declared.}

Provenance and peer review Not commissioned; internally peer reviewed.

(c) European Association of Hospital Pharmacists (unless otherwise stated in the text of the article) 2017. All rights reserved. No commercial use is permitted unless otherwise expressly granted.

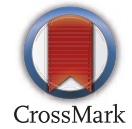

To cite Lewis P. Eur J Hosp Pharm 2017;24:314. Published Online First 9 October 2017

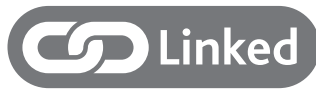

- http://dx.doi.org/10.1136/ejhpharm-2016-001165

Eur J Hosp Pharm 2017;24:314.

doi:10.1136/ejhpharm-2017-001395

\section{REFERENCES}

1 Falconer N, Nand S, Liow D, et al. Development of an electronic patient prioritization tool for clinical pharmacist interventions. Am J Health Syst Pharm 2014;71:311-20.

2 Cottrell R, Caldwell M, Jardine G. Developing and implementing a pharmacy risk screening tool. Eur J Hosp Pharm 2013;71.

3 Hickson RP, Steinke DT, Skitterall C, et al. Evaluation of a pharmaceutical assessment screening tool to measure patient acuity and prioritise pharmaceutical care in a UK hospital. Eur J Hosp Pharm 2017;24:74-9.

4 Office of the chief pharmaceutical officers MD. NHS England transformation of 7 day clinical pharmacy services in acute hospitals: NHS England, 2016. 